\title{
Seeing stars in a big way
}

\section{The Gemini project typifies the growth of astronomy into 'big science'.}

\section{Giant Telescopes: Astronomical Ambition and the Promise of Technology \\ by W. Patrick McCray \\ Harvard University Press: 2004. 376 pp. \\ $\$ 45, £ 29.95, € 41.50$}

\section{Sidney C. Wolff}

In the past 25 years, the number and size of ground-based optical and infrared telescopes has increased at an unprecedented rate. Giant Telescopes by science historian W. Patrick McCray describes part of this rise, the Gemini project, which led to the creation of two telescopes with mirrors that are 8 metres in diameter, the first in Hawaii and the second in Chile. McCray focuses on US involvement in the project, but Britain, Canada, Argentina, Brazil, Chile and Australia are also partners in Gemini.

The book begins with the completion in 1948 of the 5-metre telescope at Palomar Mountain, California. It then describes the controversy that surrounded - and still surrounds - the establishment and definition of the role of the US National Optical Astronomy Observatory (NOAO). Should it provide flagship facilities to support cuttingedge research? Or should it merely be, as many astronomers at élite universities have argued, one of several equivalent observatories with the responsibility of providing observing time to astronomers at institutions that do not have their own facilities? Even now, nearly $80 \%$ of telescope surface available to US astronomers is owned by university and private observatories, which restrict access primarily to their own staff.

Giant Telescopes discusses the technical breakthroughs in developing lightweight mirrors that enabled the construction of these telescopes. Three different technologies were developed in the 1980s: thin, flexible meniscus mirrors; stiffer, honeycombed borosilicate mirrors; and segmented mirrors. All of these have now been incorporated in telescopes that deliver excellent image quality. McCray summarizes some of the scientific questions that stimulated the worldwide investment in new telescopes.

The efforts of the NOAO to initiate a giant-telescope project are then described. After many false starts, the NOAO successfully nurtured the Gemini project and identified international partners. The Gemini telescope in Hawaii had first light in 1999, the one in Chile a year later, and both telescopes are now operating successfully.

McCray has relied on written documentation and interviews with the key players in the Gemini programme to tell his story. The

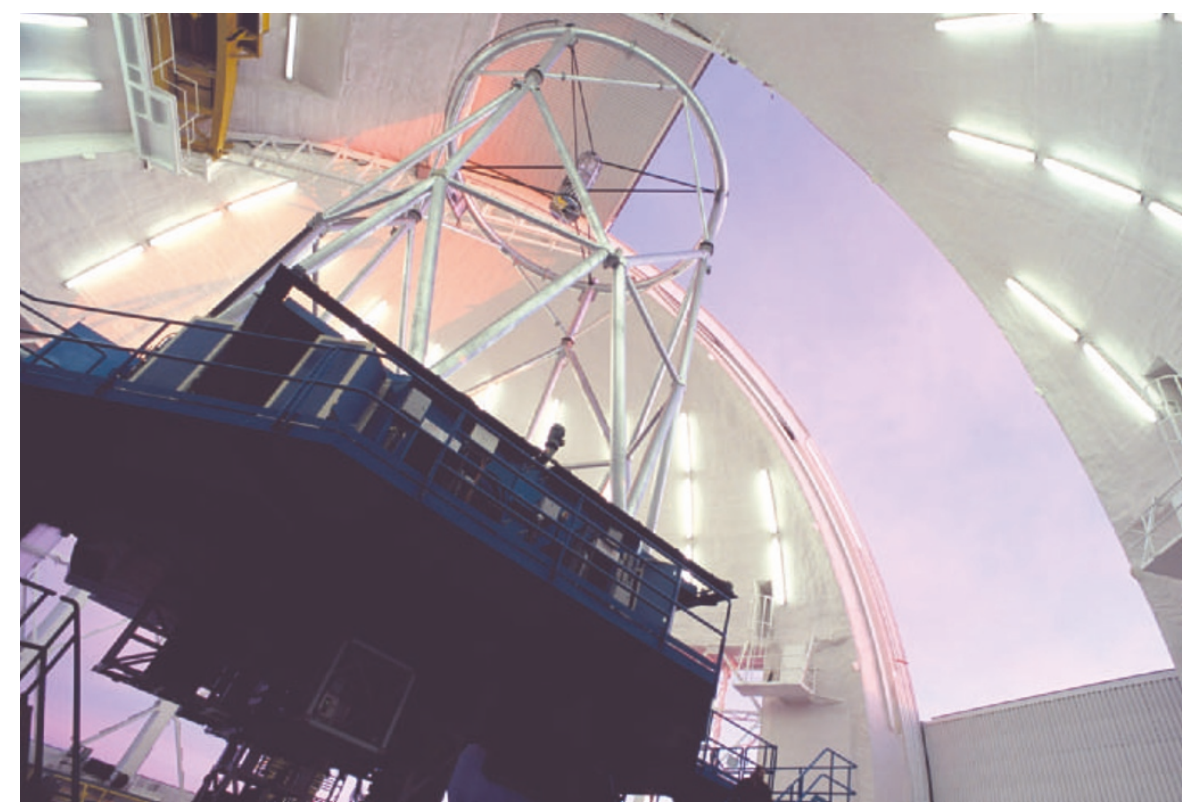

A clear vision: the Gemini South telescope in Chile is part of a large international project.

interviews were conducted while memories were still fresh, so the book is a valuable record of a critical period of astronomical development. Not only was a new generation of telescopes under construction, but astronomy was making the transition to becoming 'big science'. It was moving from an age when instruments and telescopes were built by small groups led by innovative astronomers who made real-time decisions about what to observe to a time of projects costing hundreds of millions of dollars that required large teams with specialized engineering, software and management expertise. McCray examines the social, fiscal and institutional forces that influenced postwar astronomy and other sciences that were dependent on ever-increasing financial investment.

As a participant in many of the events described, I found the book to be accurate overall. McCray does a good job of representing the various points of view about controversial issues without making value judgements, and his lack of bias makes this a particularly useful record. Equally importantly, the book is quite simply a good read. The explanations of both technical and scientific issues are clear and correct, and the book can be easily understood by anyone with an interest in either astronomy or the sociology and politics surrounding bigscience projects.

US ground-based astronomy has historically been dependent on a pluralistic approach involving private philanthropy, universities and federal funding. Europe, in contrast, has chosen to unify its efforts through the European Southern Observatory. Which will prove to be the best approach now that astronomers are initiating technical studies for still larger telescopes? Europe has set as its goal a 50-100-metre facility, whereas US astronomers are designing 20-30-metre telescopes. Are the more modest US aspirations a correct assessment of what is technically feasible or a sign that the fragmented US community has finally ceded leadership in ground-based astronomy to Europe?

There are two competing designs in the United States. One is based on compound, segmented mirrors, like those on the 10metre Keck telescopes on Mauna Kea in Hawaii. The other would make use of seven separate mirrors, each 8.4 metres in diameter, on a single mount. This competition is eerily reminiscent of the shoot-out in the mid-1980s, described by McCray, between two approaches to building a 16-metre telescope for the US community. In the end, that telescope was never built, in part because of the failure of US astronomers to coalesce around a single project. There are many lessons in McCray's book for those who would avoid repeating history.

Sidney C. Wolff is at the National Optical Astronomy Observatory, 950 North Cherry Avenue, Tucson, Arizona 85719, USA.

\section{More on telescopes}

A Visitor's guide to the Kitt Peak

\section{Observatories}

Leslie Sage \& Gail Aschenbrenner

Cambridge University Press, 2004

$\$ 15, £ 12.99$ 